\title{
Article \\ Feasibility and Effect of a Wearable Motion Sensor Device in Facilitating In-Home Rehabilitation Program in Patients after Total Knee Arthroplasty: A Preliminary Study
}

\author{
Yu-Pin Chen ${ }^{1,2} \mathbb{D}$, Chung-Ying Lin ${ }^{3} \mathbb{D}$, Yi-Jie Kuo ${ }^{1,2}$ and Oscar Kuang-Sheng Lee 4,5,* $^{4}$ \\ 1 Department of Orthopedics, Wan Fang Hospital, Taipei Medical University, Taipei 116, Taiwan; \\ 99231@w.tmu.edu.tw (Y.-P.C.); benkuo5@gmail.com (Y.-J.K.) \\ 2 Department of Orthopedics, School of Medicine, College of Medicine, Taipei Medical University, \\ Taipei 110, Taiwan \\ 3 Institute of Allied Health Sciences, College of Medicine, National Cheng Kung University, Tainan 701, Taiwan; \\ cylin36933@gmail.com \\ 4 Institute of Clinical Medicine, National Yang Ming Chiao Tung University, Taipei, 112, Taiwan \\ 5 Department of Orthopedics, China Medical University Hospital, Taichung 404, Taiwan \\ * Correspondence: oscarlee9203@gmail.com
}

check for updates

Citation: Chen, Y.-P.; Lin, C.-Y.; Kuo, Y.-J.; Lee, O.K.-S. Feasibility and Effect of a Wearable Motion Sensor Device in Facilitating In-Home Rehabilitation Program in Patients after Total Knee Arthroplasty: A Preliminary Study. Appl. Sci. 2022, 12, 2433. https://doi.org/10.3390/ app12052433

Academic Editor:

Subhas Mukhopadhyay

Received: 5 January 2022

Accepted: 23 February 2022

Published: 25 February 2022

Publisher's Note: MDPI stays neutral with regard to jurisdictional claims in published maps and institutional affiliations.

Copyright: (c) 2022 by the authors. Licensee MDPI, Basel, Switzerland. This article is an open access article distributed under the terms and conditions of the Creative Commons Attribution (CC BY) license (https:// creativecommons.org/licenses/by/ $4.0 /)$.
Featured Application: Telerehabilitation using a wearable motion sensor device for patients and health systems.

\begin{abstract}
Postoperative home-based rehabilitation programs are essential for facilitating functional recovery after total knee replacement (TKA). This study aimed to verify the feasibility of applying a wearable motion sensor device (MSD) to assist patients in performing home-based exercises after TKA. The interrater reliability of the measurement for knee mobility and the time spent completing the 5-times sit-to-stand test (5TSST) by two experienced physicians and using the MSD in 12 healthy participants was first assessed. A prospective control trial was then conducted, in which 12 patients following TKA were allocated to two groups: the home-based exercise group and the MSD-assisted rehabilitation group. Changes in knee range of motion, pain, functional score, performance, and exercise completion rates were compared between the groups over two months of follow-up. MSDmeasured knee mobility and 5TSST exhibited excellent reliability compared with the physician measurements. Furthermore, patients in the MSD-assisted rehabilitation group reported higher training compliance than participants in the home-based exercise group, which led to better outcomes in the knee extension angle and maximal and average angular velocity in 5TSST. MSD-assisted home-based rehabilitation following TKA is a feasible treatment model for telerehabilitation because it enhances patients' compliance to training, which improves functional recovery.
\end{abstract}

Keywords: knee replacement; home-based exercise; telerehabilitation; motion sensors

\section{Introduction}

Osteoarthritis (OA) of the knee refers to the wear of knee joint cartilage, causing pain and a limited range of motion (ROM) among patients. Knee OA is one of the main reasons for disability among older adults [1], and nearly one-tenth of the population aged over 60 experience pain and discomfort caused by knee OA [2]. Total knee arthroplasty (TKA) is currently the most effective treatment for improving long-term knee function and quality of life among patients with end-stage knee OA [3]. The rates of TKA have doubled in the United States in recent years, with advances in the technique and prosthesis design indicating an increasing demand for healthcare financing and postoperative clinical management [4].

Physiotherapy, such as hospital-based rehabilitation [5], outpatient-guided physical therapy [6], or cross-team multidisciplinary rehabilitation [7], is effective for alleviating 
postoperative discomfort and accelerating functional recovery in patients after TKA [8,9]. Protocols to improve post-surgical recovery have led to a decrease in the length of hospital stay among patients after arthroplasty [10]. The time for inpatient rehabilitation after TKA is thus decreased, highlighting the need for guidance on home-based rehabilitation for patients. However, the provision of post-hospital rehabilitative care is highly variable, and the barriers to accessing therapists and the financial burden of rehabilitation services should be considered, given the demand for high-quality rehabilitation services following TKA [11]. In-home exercise programs are easily accessible self-training programs that can be performed by patients, and they have similar effectiveness to inpatient rehabilitation programs following TKA [12]. However, the success of functional recovery depends on the patients' training compliance and in-home exercise correctness. Research has demonstrated that physical activity does not increase in patients following joint replacement [13], and only a quarter of patients with OA comply with their exercise program [14]. Developing more reliable methods to maximize the effectiveness of home-based physiotherapy remains challenging.

Techniques for detecting body motions could be employed to monitor and rehabilitate disabled patients, offering independent training that has advantages over traditional rehabilitation services [15]. Inertial measurement units (IMUs), including accelerometers and gyroscopes, have been extensively used in technology-assisted rehabilitation with sufficient accuracy [16-18]. The IMU-based motion tracking systems, which can be used to reconstruct the position or the orientation of the body they are attached to, have the advantage of cost-effectiveness and wearability, allowing for long-lasting tracking of the user motion in situated environments [19]. In our previous study, we developed a wearable motion sensor device (MSD) utilizing the wearable IMU-based sensors to trace the shoulder's angular motion to assist with in-home shoulder exercises for patients with adhesive capsulitis [20]. In addition, the design of the MSD was integrated with interactive mobile apps through wireless telecommunication technology to increase patient-provider interaction, thereby supporting the feasibility of image- and sensor-based telerehabilitation in the treatment of adhesive capsulitis. Our results demonstrated preliminary success in increasing patients' adherence to daily rehabilitation programs, which facilitated shoulder functional recovery. However, further investigation is required to assess whether the use of a wearable MSD in telerehabilitation can increase patients' engagement in the in-home exercise program, thereby improving knee function after TKA.

Focusing on the potential benefits of MSD in the post-TKA telerehabilitation scenario, this study replicated the previous experience of using the MSD for treating adhesive capsulitis to verify the feasibility of an MSD-based treatment model in assisting patients with home-based exercises after TKA. The application of the MSD in this study, which contained two wearable IMU-based sensors to trace the knee angular motion and was integrated with interactive mobile apps designed for patients and physiotherapists to follow and monitor in-home exercise progress, was anticipated to improve patient compliance with post-TKA rehabilitation exercises. We hypothesized that the use of a wearable MSD can help patients perform an in-home exercise program correctly and increase their motivation for daily exercise training, thereby improving functional outcomes after TKA.

The remainder of this paper is organized as follows. Section 2 presents the materials and methods, including the design of the MSD and the study protocols for (1) the reliability of knee mobility measurements using the MSD and (2) the feasibility of using the MSD for the post-TKA in-home rehabilitation. Section 3 provides our preliminary results and the participants' user experience of the MSD design. Section 4 discusses the effect and clinical value of the MSD and the limitations of this study. Lastly, conclusions are presented in Section 5. 


\section{Materials and Methods}

\subsection{Motion Sensor Device (MSD)}

A body-worn MSD (BoostFix wearable self-training kit, COMPAL, Taipei, Taiwan) (see Supplementary Materials Files) was employed in this study. The device comprised (1) wearable IMU-based sensors to record the ROM of the knee, (2) the mobile application Patient App for use by the patient, and (3) the mobile application Doctor App for use by therapists.

\subsubsection{Wearable IMU-Based Sensors}

The IMU-based sensors comprised six-axis microelectromechanical systems, composed of accelerometers and gyroscopes, and the sensors collected information on the angular motion of the knee of interest. These 2-sensor systems were used in the MSD to accurately record the knee's angular motion. To perform the recording, 2 sensors were strapped on the anterior midline of the distal third of the femur (i.e., $10 \mathrm{~cm}$ proximal to the upper border of the patella) and the proximal third of the tibia (i.e., $10 \mathrm{~cm}$ distal to the tibia tuberosity) (Figure 1a). An algorithm was used to convert raw data from the accelerometer and gyroscope into a quaternion. The sensors were calibrated in the position of the knee flexion at $90^{\circ}$ when the measurement began (Figure $1 \mathrm{~b}$ ). The initial calibration process involved placing the sensor on a horizontal fixture to measure the offset for each axis and to eliminate nonzero deviations. The sensors on the thigh served as a reference point, enabling raw data from the sensor on the proximal tibia to be used to construct a quaternion using the algorithm; thus, the relative angle changes on the knee joint could be converted into a structure based on the motion of the knee. The high accuracy of repeated angular measurements taken using the 2-sensor system on simulated knee motion was also validated ex vivo (see Supplementary Materials Files).

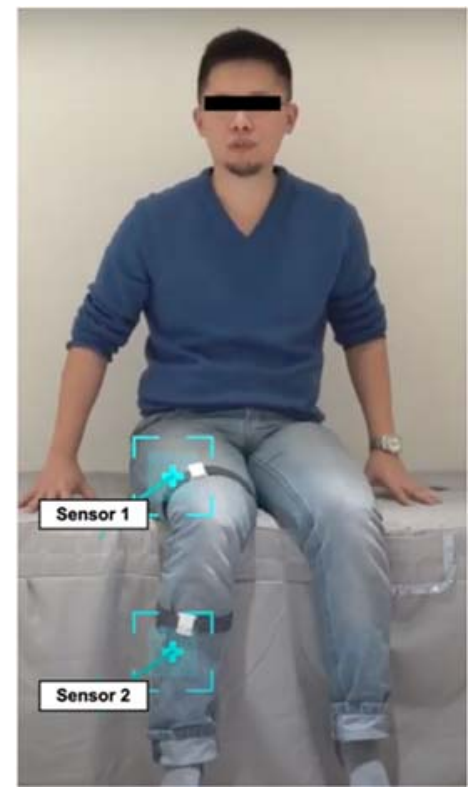

(a)

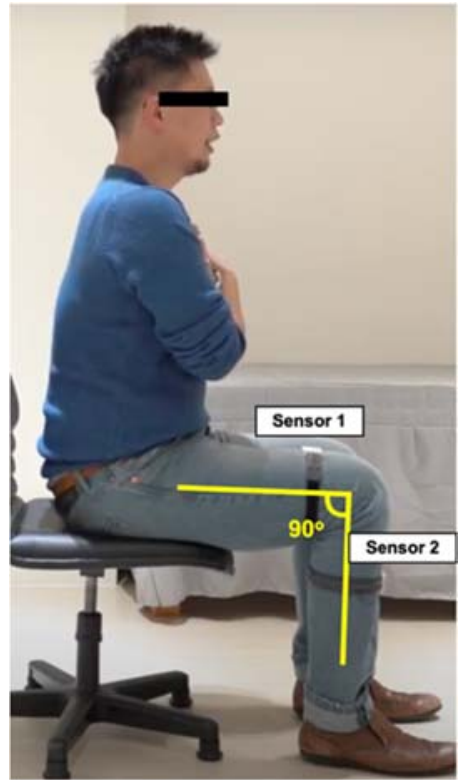

(b)

Figure 1. The position of motion sensors worn on the lower limb: (a) sensor position; (b) calibration position.

InvenSense's built-in library was utilized to setup the algorithm [21]. The fusing algorithm combined gyroscope and accelerometer measurements into a single measurement of orientation as a quaternion by drifting in the yaw component of orientation only. The application could reset the drift in yaw by setting the yaw to a specified angle at any time. The algorithm also contained functions for converting this quaternion into a rotation matrix and Euler angles. In addition, the algorithm provided a measurement of linear 
acceleration (equal to the accelerometer measurement with the $1 \mathrm{~g}$ of gravity removed) and Earth acceleration (a measurement of linear acceleration in the Earth coordinate frame). The example of pseudo-code was provided in Supplementary Materials Files.

\subsubsection{A Mobile Phone App for Patients}

An app was designed for use on mobile phones. The app contained a feature for measuring the angle of knee flexion and extension. Furthermore, the mobile app offered a feature that recorded the time required to complete a 5-times sit-to-stand test (5TSST), which is an objective performance-based measurement for patients following TKA [22,23]. The maximal angular velocity and average angular velocity when rising from a chair are measured in 5TSST, which represents the strength and endurance of the quadriceps muscle [23]. The strength and endurance of the quadriceps muscles can also be calculated based on the relative angular change with time using the accelerometers on the 2 sensors. Furthermore, the mobile app could generate historical records of knee angular measurements, the performance in 5TSST, and exercise completion rates (ECRs) as well as provide detailed instructions for daily knee exercises (Figure 2). The Patient App provides instructions for ten sets of home-based knee exercises for training, which are based on the in-home exercise program designed by Buhagiar M.A. et al. (Figure 3 and Supplementary Materials Files) [12].

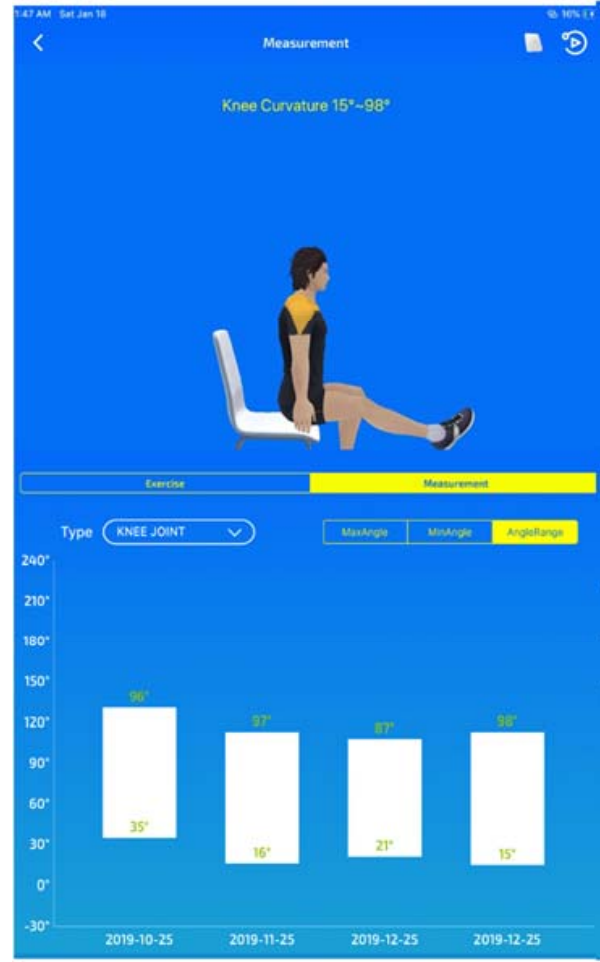

(a)

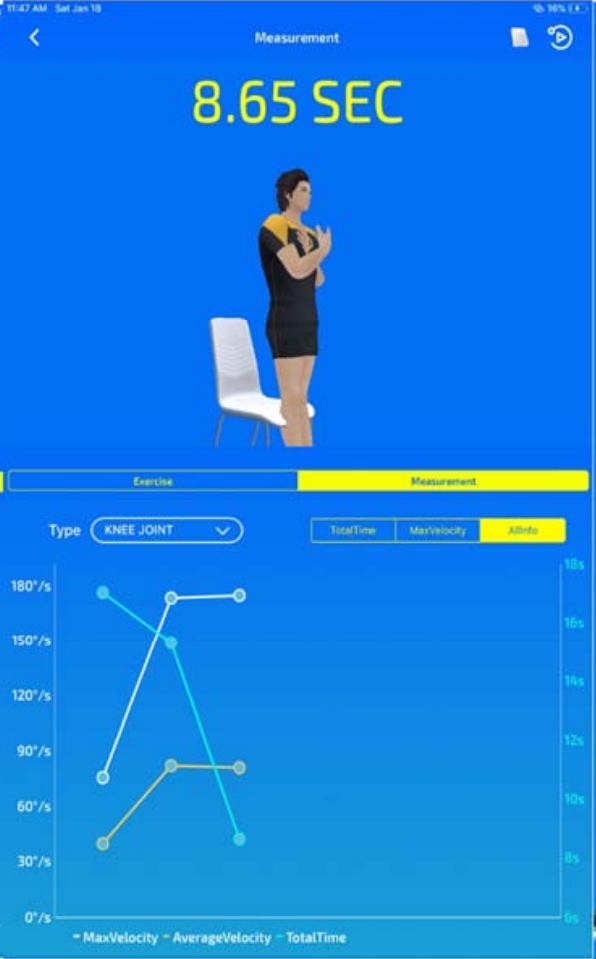

(b)

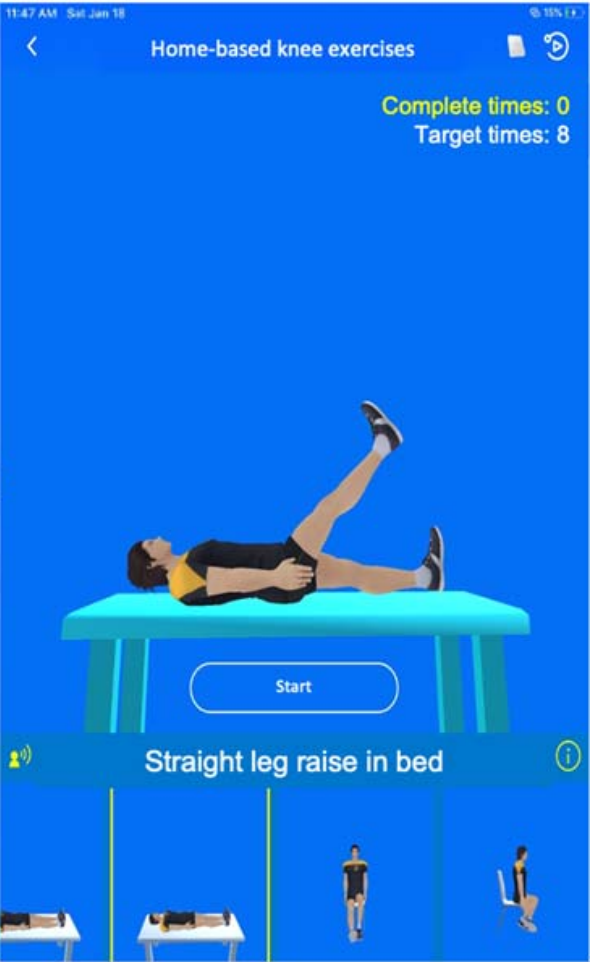

(c)

Figure 2. Mobile app for patients: (a) historical records of knee flexion and extension; (b) historical records of 5TSST; (c) daily home-based exercise tasks.

Through the mobile app, daily exercise tasks assigned by the supervising physiotherapist or physicians were provided to patients. The app demonstrated a three-dimensional (3D) avatar to mirror the user's knee motion while performing the assigned exercise (Figure 3). Each user had an account on the Patient App to access their records, including daily progress and ECR (Figure 2a,b). 


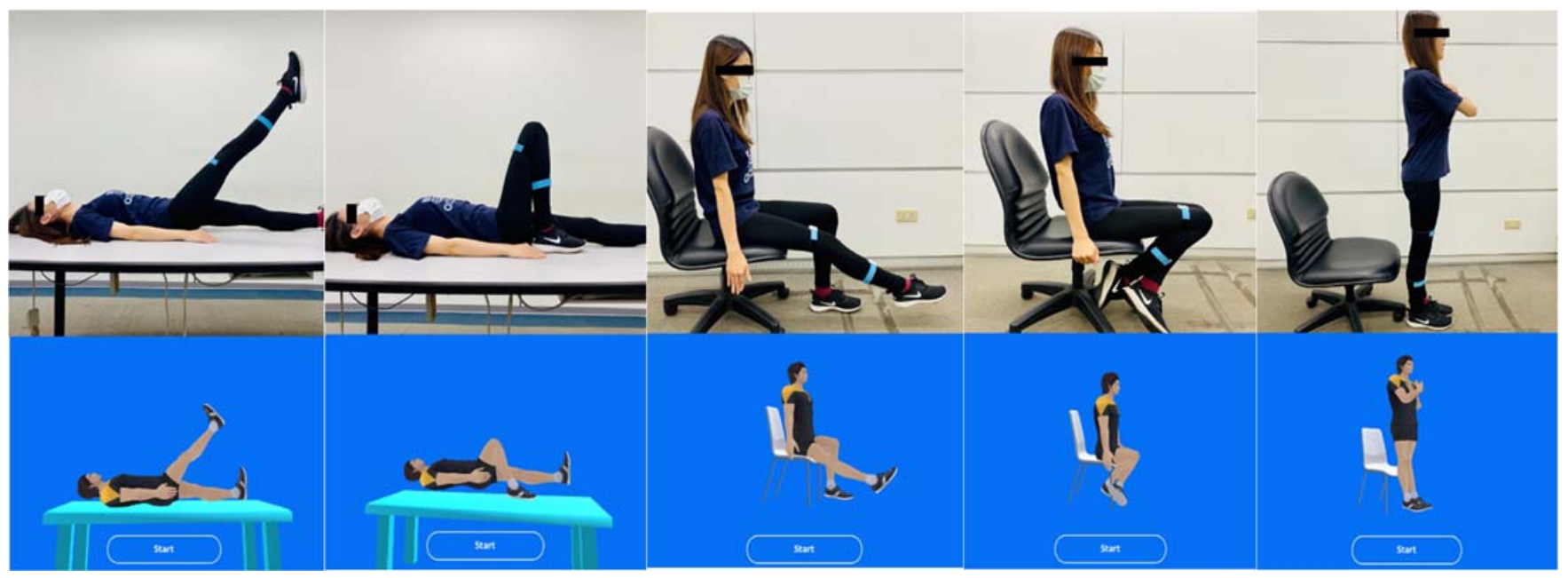

(a)

(b)

(c)

(d)

(e)

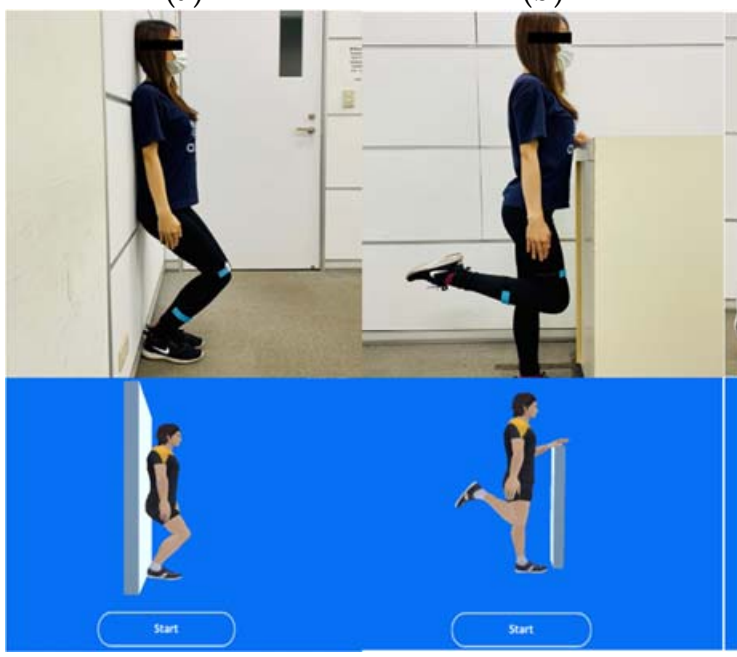

(f)

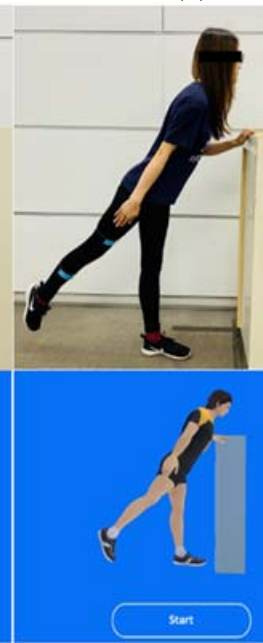

(h)

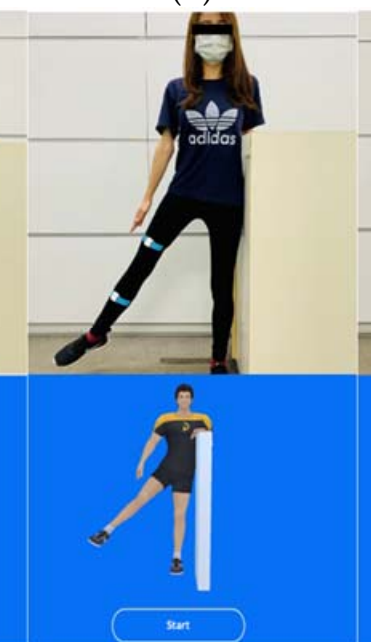

(i)

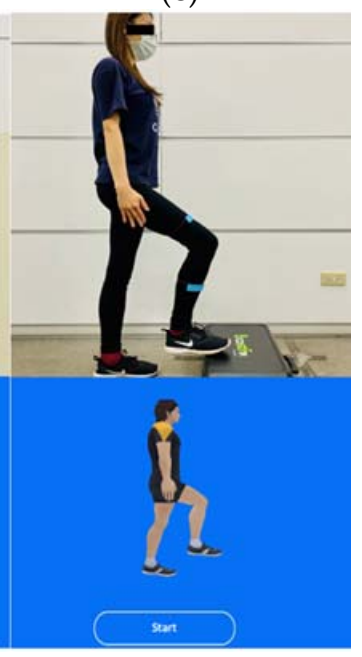

(j)

Figure 3. Sets of home-based knee exercises displayed on the mobile app and demonstrated by participants: (a) straight leg raise in bed; (b) knee flexion in bed; (c) sitting knee stretch; (d) sitting knee flexion; (e) sit-to-stand; (f) semi-squat on wall; (g) standing knee flexion; (h) standing hip extension; (i) standing hip abduction; (j) forward step-ups.

\subsubsection{A Mobile Pad App for Physiotherapists and Physicians}

The Doctor App was designed for physiotherapists and physicians to use on a mobile pad. The app provided each patient's information, including the latest knee angular measurements and ECR for the previous week (Figure 4a). Based on the individual status of the target knee, physicians could assign personalized daily home-based exercise (HE) with adjustable targeted angles, numbers of repetitions, and holding times for each patient (Figure $4 b$ ).

\subsection{Study Protocol}

This study comprised two investigations: (1) the reliability of knee ROM measurements and the time spent completing the 5TSST using the MSD and (2) the feasibility and user experience of using the MSD for the post-TKA in-home rehabilitation. The Ethics Committee of Taipei Medical University approved the entire protocol and instrumentation (TMU-JIRB N201802034). All participants consented to participate in the study and the publication of the data. 


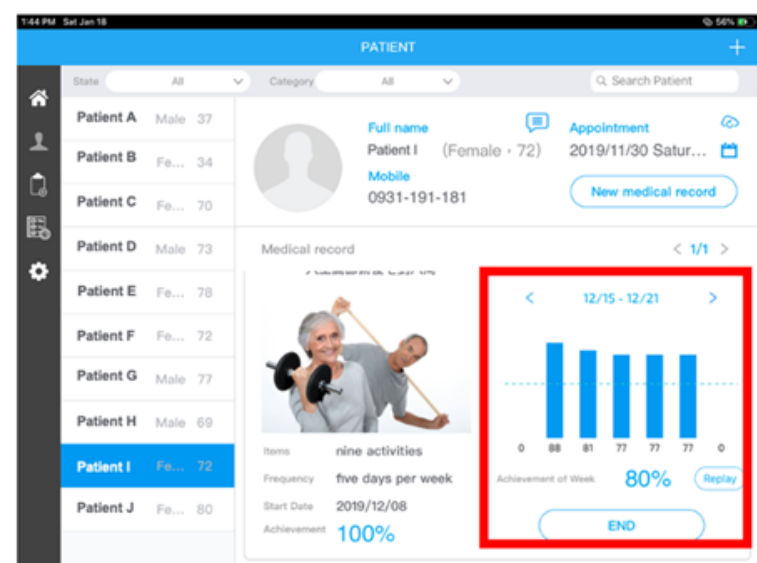

(a)

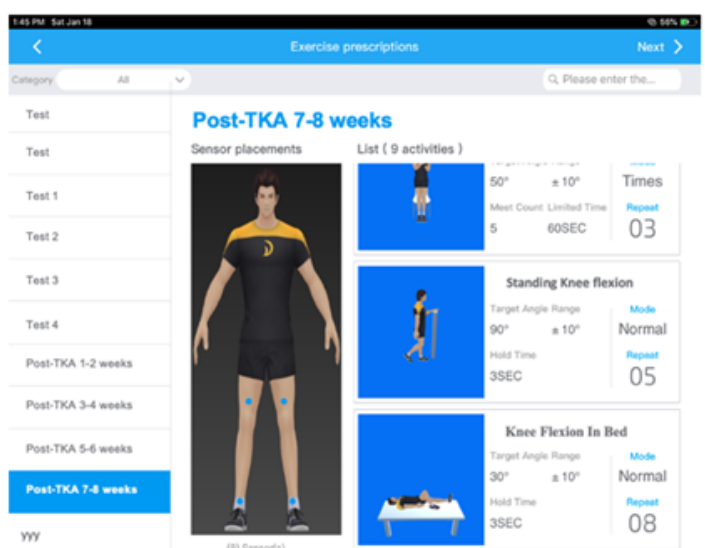

(b)

Figure 4. Mobile app for physiotherapists and physicians: (a) the daily and weekly ECR of each patient is displayed with patient information; (b) doctors assign daily exercises with specified targeted angles, holding times, and numbers of repetitions.

2.2.1. First Investigation: Evaluating the Reliability of Knee ROM Measurements and the Measurements of Time Spent for Completing 5TSST Using the MSD

A total of 12 healthy participants, aged 20-70 years and without reported discomforts or limited ROM of the knee within the preceding year, were prospectively enrolled to determine knee angular motion and time spent for completing 5TSST.

Angle Measurements

Each volunteer wore the MSD on the right leg and was seated in the middle of the chair with the back straight, without resting on the backrest, feet flat on the ground, and arms crossed over the chest (Figure 1b). Participants were instructed to discretionarily flex the knee in the three targeted range of knee flexion angles, which were $<60^{\circ}$, approximately $90^{\circ}$, and $>90^{\circ}$. The knee angular motion attempts were first assessed using the MSD. Two examiners (a physical therapist and an experienced orthopedic surgeon) were blinded to the MSD angle measurements and then measured knee ROM using a universal goniometer.

\section{Time for Completing 5TSST}

Each volunteer was seated in the middle of a calf-height chair, as illustrated in Figure $1 b$, and was instructed to rise to fully stand and then return to a fully seated position as fast as possible 5 times. The time required to complete 5 sets was recorded using the MSD and by the researchers.

2.2.2. Second Investigation: Determining the Feasibility and User Experience of Using the MSD for the Post-TKA In-Home Rehabilitation

A total of 12 patients aged over 60 years who had received TKA using posteriorstabilizing instruments for advanced knee OA were enrolled. Patients were excluded if they (1) received TKA for a pathology other than OA; (2) had a history of knee replacement on the opposite knee; (3) had severe complications after TKA, including symptomatic deep vein thrombosis, fracture, and infection; (4) lived alone without family support for in-home knee exercise training; (5) had a history of limb disorders that might have interfered with the knee angular motion, including severe limb fracture, limb deformity, or neurovascular disorders; and (7) were unwilling to receive an intervention or participate in the trial.

After the surgical wound had healed in the second week following TKA, patients were allocated into 2 intervention groups: 6 patients were assigned to the HE group and 6 were assigned to the motion sensor-assisted rehabilitation (MAR) group; patients were assigned the MAR group based on their willingness to use and familiarity with mobile apps. The patients in both groups received 2 months of in-home rehabilitation. 
Before beginning the exercise program, patients in the HE group received comprehensive instructions on knee rehabilitation after TKA from an experienced orthopedic surgeon. The standard protocol of HEs is available in Multimedia Supplementary Materials Files. These patients were instructed to follow the standard HE protocol but individualized modifications were permitted based on health professionals' judgment. An orthopedic surgeon reviewed the progress of each patient every 2 weeks, provided advice, and modified the exercise program based on patient compliance and the standard protocol.

Before they began the in-home exercise program, all patients in the MAR group received detailed instructions on operating the MSD, and the Patient App was installed on their mobile phones. The orthopedic surgeon used the Patient App to assign daily HEs to each patient based on the standard protocol (see Supplementary Materials Files). Furthermore, through the mobile app, the orthopedic surgeon individually assigned each set of exercises with a corresponding target angle to patients in the MAR group based on the angle that patients could successfully achieve (Figure $4 b$ ). Achievement of the assigned exercise was recorded as successful completion of the exercise, which was used to calculate the daily ECR on the mobile app (Figure 4a).

\section{Outcome Measurement}

Face-to-face interviews were performed to collect basic demographic data, including age, sex, body mass index, and site of the operated limb for each patient who had received TKA. The maximal angle of knee flexion and extension of the operated knee, the time required to complete 5TSST, and the maximal and average angular velocity when rising from a chair in 5TSST were assessed and recorded using the MSD at baseline (i.e., 2 weeks following TKA) and in each month for 2 months of the in-home rehabilitation program. All patients completed questionnaires that allowed relevant metrics at baseline and at 1 and 2-month follow-up to be evaluated by an independent physical therapist who was not involved in treating any of the patients and was blinded to treatment allocation. The questionnaires were based on the visual analog scale (VAS) of pain severity and the Western Ontario and McMaster University Osteoarthritis Index (WOMAC). The ECR was recorded monthly from participants' reports and the MSDs in the MAR group.

Instruments

\section{Measurement of knee function}

The self-reported WOMAC consists of three subscales (pain, stiffness, and physical function) comprising 24 items. The WOMAC has promising psychometric properties [24]. A higher WOMAC indicates worse knee function.

\section{Measurement of pain}

The VAS is an instrument for measuring pain intensity [25]. Patients were asked, "On a scale of 0 to 10 , how severe was the worst pain on your operated knee that you have experienced within the last week?"

\section{Measurement of ECR}

The ECR was calculated from the MSD data by dividing the number of exercises completed daily by the number of assigned daily exercise tasks. The monthly ECR was the average of the daily ECRs over the entire month. The monthly patient reported ECRs were obtained by asking each patient, "On a scale of $0 \%$ to $100 \%$, what was your average exercise completion rate in the previous month?"

\section{User Experience}

After 2 months of the in-home rehabilitation program, all patients in the MAR group were contacted via telephone for a 20-min interview on their experience of the MSD design. Closed- and open-ended questions were used to evaluate their actual use of the device and obtain feedback and detailed suggestions. 
The following 4 items were assessed as closed questions on a $0-10$ scale (Cronbach's alpha $=0.93$ ):

1. Helpfulness of the MSD in assisting with rehabilitation: "On a scale of 0 to 10 (0 indicating the least helpful and 10 indicating the most helpful), how would you rate the helpfulness of the wearable motion sensor device in assisting your home-based exercises after knee replacement?"

2. Ease of operability for the MSD: "On a scale of 0 to 10 (0 indicating the most difficult and 10 means the easiest), how would you rate the ease of operability for the motion sensor device in your daily exercise?"

3. Satisfaction with the app design: "On a scale of 0 to 10 ( 0 indicating the least satisfied and 10 means the most satisfied), how satisfied are you with the app's design and visual appeal?"

4. Interests for future use: "On a scale of 0 to 10 (0 indicating the least interested and 10 means the most interested), how interested would you be in using the device after it is fully developed in assisting your future rehabilitation if needed?"

After finishing all interviews, the research team summarized the findings and forwarded them to participants for confirmation and further feedback, if any.

\subsection{Statistical Analysis}

The intraclass correlation coefficient (ICC) was used to calculate the interrater reliability of the outcomes of knee ROM and the 5TSST time measured using the MSD and by the 2 experienced examiners. The ICC was obtained by performing a 2-way analysis of variance in a random-effects model. ICCs of $>0.8$ and $>0.9$ indicate good and excellent reliability, respectively [26].

The Mann-Whitney $U$ test was used to compare the HE and MAR groups due to the small sample size. The Wilcoxon signed-rank test was used for within-group comparisons of dependent variables at follow-up. Descriptive statistics for participant characteristics and generalized estimating equations were used to compare the improvements in several outcomes between the 2 groups (knee active ROM, VAS, WOMAC, time for 5TSST, and the maximal and average angular velocity in 5TSST). All outcomes were analyzed using generalized estimating equations with the restricted maximum likelihood estimation. Furthermore, all generalized estimating equations controlled for the effects of time during follow-up. IBM SPSS 23.0 (IBM corp. Armonk, NY, USA) was used for all data analyses, and $p \leq 0.05$ was considered statistically significant.

\section{Results}

Table 1 presents the results for knee angular measurements in different ranges of angle and the time required to perform 5TSST among healthy participants, which were measured using the MSD and by the two examiners and were used to assess the reliability of MSD measurements. The interrater reliability for the angular measurement of knee flexion in three range of angles, as assessed using the MSD and by the two examiners, was considered good to excellent (ICC range: 0.862-0.934). The interrater reliability for the time required to perform 5TSST between the MSD and the two examiners was also excellent (ICC: 0.996).

Table 2 presents the patient demographics, which did not differ significantly at baseline between the two groups. After two months of rehabilitation training, patients in both the MAR and HE groups exhibited significant improvements from baseline in all parameters, except pain on the VAS (Table 3). However, patients in the MSD group demonstrated significantly better recovery of the knee maximal extension angle $(p=0.04)$ and performance in the 5TSST time and maximal and average angular velocity ( $p=0.04,0.05,0.03$, respectively) at the 2-month follow-up (Table 3). 
Table 1. Interobserver reliability between angle measurements and measurements of the time for performing 5 -times sit-to-stand test obtained by different examiners.

\begin{tabular}{|c|c|c|c|c|c|}
\hline \multirow[b]{2}{*}{ 12. Volunteers } & \multicolumn{4}{|c|}{ Knee Flexion within the Targeted Range of Angle } & \multirow{2}{*}{$\begin{array}{c}\text { Time Spent for } \\
\text { Completing 5TSST } \\
(n=12)\end{array}$} \\
\hline & $\begin{array}{c}\text { Angle }<60^{\circ} \\
(n=12)\end{array}$ & $\begin{array}{c}\text { Angle } \approx 90^{\circ} \\
(n=12)\end{array}$ & $\begin{array}{c}\text { Angle }>90^{\circ} \\
(n=12)\end{array}$ & $\begin{array}{l}\text { Overall } \\
(n=36)\end{array}$ & \\
\hline Examiner 1 & $34.7^{\circ} \pm 9.7^{\circ}$ & $89.6^{\circ} \pm 5.3^{\circ}$ & $122.8^{\circ} \pm 10.4^{\circ}$ & $82.4^{\circ} \pm 37.8^{\circ}$ & $11.2 \pm 1.7$ \\
\hline Examiner 2 & $37.2^{\circ} \pm 8.3^{\circ}$ & $92.3^{\circ} \pm 7.1^{\circ}$ & $124.2^{\circ} \pm 10.4^{\circ}$ & $84.6^{\circ} \pm 37.4^{\circ}$ & $11.2 \pm 1.5$ \\
\hline Motion sensor & $35.0^{\circ} \pm 9.4^{\circ}$ & $92.9^{\circ} \pm 8.6^{\circ}$ & $123.5^{\circ} \pm 11.6^{\circ}$ & $83.8^{\circ} \pm 38.4^{\circ}$ & $11.3 \pm 1.6$ \\
\hline ICC among examiners & 0.924 & 0.862 & 0.934 & 0.996 & 0.996 \\
\hline
\end{tabular}

Notes: ICC: intraclass correlation coefficient; 5TSST: 5-times sit-to-stand test.

Table 2. Comparison of patient demographics between groups.

\begin{tabular}{|c|c|c|c|}
\hline & MAR Group $(n=6)$ & HE Group $(n=6)$ & $p$-Value \\
\hline Age & $70.3 \pm 2.8$ & $70.2 \pm 5.7$ & 0.95 \\
\hline Body mass index & $26.9 \pm 2.6$ & $28.6 \pm 4.3$ & 0.52 \\
\hline \multicolumn{4}{|l|}{ Sex } \\
\hline male & $3(50.0 \%)$ & $2(33.3 \%)$ & \multirow{2}{*}{1} \\
\hline female & $3(50.0 \%)$ & $4(66.7 \%)$ & \\
\hline \multicolumn{4}{|c|}{ Education } \\
\hline Elementary & $5(83.3 \%)$ & $3(50.0 \%)$ & \multirow{3}{*}{0.22} \\
\hline Senior high & $1(16.7 \%)$ & $2(33.3 \%)$ & \\
\hline Bachelor degree and higher & $0(0.0 \%)$ & $1(16.7 \%)$ & \\
\hline \multicolumn{4}{|c|}{ Operated side } \\
\hline left & $2(33.3 \%)$ & $4(66.7 \%)$ & \multirow{2}{*}{0.57} \\
\hline right & $4(66.7 \%)$ & $2(33.3 \%)$ & \\
\hline \multicolumn{4}{|c|}{ Baseline condition (before intervention) } \\
\hline Maximal knee extension $\left(^{\circ}\right)$ & $28.2 \pm 8.6$ & $24 \pm 13.7$ & 0.40 \\
\hline Maximal knee flexion $\left({ }^{\circ}\right)$ & $98.2 \pm 9.6$ & $93.5 \pm 8.8$ & 0.62 \\
\hline VAS & $3.7 \pm 1.2$ & $5.2 \pm 1.7$ & 0.11 \\
\hline WOMAC & $36.5 \pm 15.5$ & $41.7 \pm 15.7$ & 0.58 \\
\hline \multicolumn{4}{|l|}{ 5TSST } \\
\hline Total time spending (s) & $22.2 \pm 9.2$ & $27.1 \pm 11.7$ & 0.42 \\
\hline Maximal angular velocity $(\% / s)$ & $100.5 \pm 35.4$ & $94.5 \pm 35.6$ & 0.78 \\
\hline Average angular velocity $(\% / \mathrm{s})$ & $45.5 \pm 14.4$ & $31.5 \pm 12.4$ & 0.10 \\
\hline
\end{tabular}

Notes: VAS: Visual Analog Scale; WOMAC: Western Ontario and McMaster University Osteoarthritis Index 5TSST: five times sit-to-stand test.

Patients in the MAR group displayed significantly better recovery of the knee maximal extension angle over time and showed higher maximal and average angular velocity in 5TSST ( $p=0.049,0.002,0.006$, respectively) than patients in the HE group (Table 4). Table 5 illustrates that patients in the MAR group had a significantly higher overall ECR as reported by the patients during the two months of rehabilitation compared with patients in the HE group $(p=0.041)$. 
Table 3. Improvements in parameters compared with baseline and between groups.

\begin{tabular}{|c|c|c|c|c|c|c|c|c|}
\hline & \multirow[b]{2}{*}{ Baseline } & \multirow{2}{*}{$\begin{array}{l}1 \text { Month } \\
\text { Follow-Up }\end{array}$} & \multicolumn{2}{|c|}{$p$-Value } & \multirow{2}{*}{\multicolumn{2}{|c|}{$\begin{array}{l}\text { 2-Month } \\
\text { Follow-Up }\end{array}$}} & \multicolumn{2}{|c|}{$p$-Value } \\
\hline & & & $\begin{array}{c}\text { Versus } \\
\text { Baseline }\end{array}$ & $\begin{array}{l}\text { Between } \\
\text { Groups }{ }^{a}\end{array}$ & & & $\begin{array}{c}\text { Versus } \\
\text { Baseline }\end{array}$ & $\begin{array}{l}\text { Between } \\
\text { Groups b }\end{array}$ \\
\hline \multicolumn{9}{|c|}{ MAR group $(n=6)$} \\
\hline $\begin{array}{l}\text { Maximal knee } \\
\text { extension }\left(^{\circ}\right)\end{array}$ & $28.2 \pm 8.6$ & $13.0 \pm 5.8$ & 0.03 & 0.17 & \multicolumn{2}{|c|}{$9.3 \pm 5.2$} & 0.03 & 0.04 \\
\hline $\begin{array}{c}\text { Maximal knee } \\
\text { flexion }\left({ }^{\circ}\right)\end{array}$ & $98.2 \pm 9.6$ & $110.8 \pm 14.6$ & 0.03 & 0.42 & \multicolumn{2}{|c|}{$112.7 \pm 11.8$} & 0.03 & 0.23 \\
\hline VAS & $3.7 \pm 1.2$ & $3.3 \pm 0.5$ & 0.48 & 10.00 & \multicolumn{2}{|c|}{$2.3 \pm 1.2$} & 0.23 & 0.80 \\
\hline WOMAC & $36.5 \pm 15.5$ & $17.8 \pm 11.2$ & 0.03 & 0.26 & \multicolumn{2}{|c|}{$9.0 \pm 5.2$} & 0.03 & 0.09 \\
\hline \multicolumn{9}{|l|}{ 5TSST } \\
\hline Total time (s) & $22.2 \pm 9.2$ & $14.1 \pm 7.1$ & 0.03 & 0.06 & \multicolumn{2}{|c|}{$10.7 \pm 6.2$} & 0.03 & 0.04 \\
\hline $\begin{array}{l}\text { Maximal angular } \\
\text { velocity }(\% / \mathrm{s})\end{array}$ & $100.5 \pm 35.4$ & $168.3 \pm 71.4$ & 0.03 & 0.08 & \multicolumn{2}{|c|}{$180.3 \pm 82.1$} & 0.03 & 0.05 \\
\hline $\begin{array}{l}\text { Average angular } \\
\text { velocity }(\% / \mathrm{s})\end{array}$ & $45.5 \pm 14.4$ & $75.8 \pm 29.0$ & 0.03 & 0.04 & \multicolumn{2}{|c|}{$89.7 \pm 40.7$} & 0.03 & 0.03 \\
\hline \multicolumn{9}{|c|}{ HE group $(n=6)$} \\
\hline $\begin{array}{l}\text { Maximal knee } \\
\text { extension }\left(^{\circ}\right)\end{array}$ & $24 \pm 13.7$ & $18.2 \pm 6.9$ & 0.23 & 0.17 & 16.7 & $=5.2$ & 0.12 & 0.04 \\
\hline $\begin{array}{l}\text { Maximal knee } \\
\text { flexion }\left(^{\circ}\right)\end{array}$ & $93.5 \pm 8.8$ & $101.7 \pm 6.1$ & 0.04 & 0.42 & 104.8 & \pm 3.7 & 0.05 & 0.23 \\
\hline VAS & $5.2 \pm 1.7$ & $3.3 \pm 0.5$ & 0.06 & 10.00 & $2.2=$ & 1.0 & 0.03 & 0.80 \\
\hline WOMAC & $41.7 \pm 15.7$ & $25.7 \pm 12.4$ & 0.03 & 0.26 & 16.8 & $=9.2$ & 0.03 & 0.09 \\
\hline 5TSST & & & & & & & & \\
\hline Total time (s) & $27.1 \pm 11.7$ & $20.9 \pm 10.1$ & 0.03 & 0.06 & 18.2 & $=8.3$ & 0.03 & 0.04 \\
\hline $\begin{array}{l}\text { Maximal angular } \\
\text { velocity }(\% / \mathrm{s})\end{array}$ & $94.5 \pm 35.6$ & $106.5 \pm 41.1$ & 0.05 & 0.08 & 108.0 & $=37.6$ & 0.03 & 0.05 \\
\hline $\begin{array}{l}\text { Average angular } \\
\text { velocity }(\% / \mathrm{s})\end{array}$ & $31.5 \pm 12.4$ & $38.7 \pm 11.7$ & 0.03 & 0.04 & $41.2=$ & 11.3 & 0.03 & 0.03 \\
\hline & $\begin{array}{l}\text { Notes: VA } \\
\text { 5TSST: fiv } \\
\text { based exer } \\
\text { home-base }\end{array}$ & $\begin{array}{l}\text { AS: Visual Anal } \\
\text { e times sit-to-st } \\
\text { rcise groups at } 1 \\
\text { ed exercise grou }\end{array}$ & $\begin{array}{l}\text { g Scale; WOM } \\
\text { and test; }{ }^{\text {a }} \text { Com } \\
\text { month follow-u } \\
\text { ps at 2-month f }\end{array}$ & $\begin{array}{l}\text { C: Western O } \\
\text { arison betwee } \\
\text {; b Compariso } \\
\text { llow-up. }\end{array}$ & $\begin{array}{l}\text { ontario and } 1 \\
\text { an the motior } \\
\text { on between th }\end{array}$ & $\begin{array}{l}\text { [cMaster U } \\
\text { sensor-ass } \\
\text { motion se }\end{array}$ & $\begin{array}{l}\text { niversity Osteoa } \\
\text { sisted rehabilitat } \\
\text { nsor-assisted reh }\end{array}$ & $\begin{array}{l}\text { arthritis Index } \\
\text { tion and home } \\
\text { abilitation and }\end{array}$ \\
\hline & & & & $\mathrm{B}(\mathrm{SE})$ & & & & \\
\hline Dependent Variables & & (Reference: & Baseline) & & & (Referenc & e: HE Group) & \\
\hline & $\begin{array}{l}\text { 1-Month } \\
\text { Follow-Up }\end{array}$ & $p$-Value & $\begin{array}{l}\text { 2-Month } \\
\text { Follow-Up }\end{array}$ & $p$-Value & $\begin{array}{l}\text { Group at } \\
\text { 1-Month }\end{array}$ & $p$-Value & $\begin{array}{l}\text { Group at } \\
\text { 2-Months }\end{array}$ & $p$-Value \\
\hline Maximal knee extension $\left({ }^{\circ}\right)$ & $-6.6(3.4)$ & 0.0497 & $-8.1(4.0)$ & 0.043 & $-8.5(4.5)$ & 0.057 & $-10.7(5.4)$ & 0.049 \\
\hline Maximal knee flexion $\left({ }^{\circ}\right)$ & $8.2(1.9)$ & 0.000 & $11.3(3.1)$ & 0.497 & $4.5(5.3)$ & 0.397 & $3.2(4.7)$ & 0.499 \\
\hline VAS & $-1.8(0.6)$ & 0.002 & $-3.0(0.5)$ & 0.000 & $1.5(0.7)$ & 0.450 & $1.7(1.0)$ & 0.083 \\
\hline WOMAC & $-16.0(1.6)$ & 0.000 & $-24.8(2.9)$ & 0.000 & $-2.7(4.0)$ & 0.510 & $-2.7(5.3)$ & 0.617 \\
\hline 5TSST & & & & & & & & \\
\hline Total time (s) & $-6.2(1.5)$ & 0.000 & $-8.9(1.9)$ & 0.000 & $-1.9(2.3)$ & 0.413 & $-2.6(2.4)$ & 0.279 \\
\hline
\end{tabular}


Table 4. Cont.

\begin{tabular}{|c|c|c|c|c|c|c|c|c|}
\hline \multirow{3}{*}{ Dependent Variables } & \multicolumn{8}{|c|}{$\mathrm{B}(\mathrm{SE})$} \\
\hline & \multicolumn{4}{|c|}{ (Reference: Baseline) } & \multicolumn{4}{|c|}{ (Reference: HE Group) } \\
\hline & $\begin{array}{l}\text { 1-Month } \\
\text { Follow-Up }\end{array}$ & $p$-Value & $\begin{array}{l}\text { 2-Month } \\
\text { Follow-Up }\end{array}$ & $p$-Value & $\begin{array}{l}\text { Group at } \\
\text { 1-Month }\end{array}$ & $p$-Value & $\begin{array}{l}\text { Group at } \\
\text { 2-Months }\end{array}$ & $p$-Value \\
\hline $\begin{array}{l}\text { Maximal angular } \\
\text { velocity }(\% / \mathrm{s})\end{array}$ & $12.0(4.0)$ & 0.003 & $13.5(3.9)$ & 0.000 & $55.8(17.5)$ & 0.001 & $66.3(21.1)$ & 0.002 \\
\hline $\begin{array}{l}\text { Average angular } \\
\text { velocity }(\% / \mathrm{s})\end{array}$ & $7.2(1.6)$ & 0.000 & $9.7(2.0)$ & 0.000 & $23.2(8.3)$ & 0.005 & $34.5(12.5)$ & 0.006 \\
\hline
\end{tabular}

Table 5. Exercise completion rate reported by the participants and MSD.

\begin{tabular}{|c|c|c|c|c|}
\hline \multirow[b]{2}{*}{ Exercise Completion Rate } & \multicolumn{2}{|c|}{ MAR Group } & \multirow{2}{*}{$\begin{array}{c}\text { HE Group } \\
\text { Reported by } \\
\text { Participants }\end{array}$} & \multirow[b]{2}{*}{$p$-Value } \\
\hline & $\begin{array}{l}\text { Recorded by Motion } \\
\text { Sensor Device }\end{array}$ & $\begin{array}{l}\text { Reported by } \\
\text { Participants }\end{array}$ & & \\
\hline 1 month follow-up (\%) & $77.7 \pm 8.4$ & $80.0 \pm 8.6$ & $41.7 \pm 8.7$ & 0.041 \\
\hline 2 month follow-up (\%) & $83.4 \pm 8.9$ & $85.0 \pm 7.6$ & $48.3 \pm 7.0$ & 0.026 \\
\hline Overall (\%) & $80.6 \pm 8.2$ & $82.5 \pm 7.7$ & $45.0 \pm 7.2$ & 0.041 \\
\hline
\end{tabular}

The mean scores of the participants in the MAR group for the user experience related items of device helpfulness, ease of operability, satisfaction with app design, and interest in future use were $8.5 \pm 1.0$ (range: $7-10$ ), $5.0 \pm 1.4$ (range: $3-7$ ), $7.7 \pm 0.8$ (range: 7-9), and $5.8 \pm 1.9$ (range: $3-8$ ), respectively. Participants' feedback for the overall MSD design is summarized in Table 6.

Table 6. Participant feedback for the overall MSD design.

1. This device helped me correctly and easily do daily exercises at home with a clear goal.

2. Because the doctor could trace my daily progress and interacted with me, I worked diligently to complete the assigned exercise tasks every day. I had no excuse for slacking.

3. My daily exercise performance records can be easily found on the app, helping me foster a sense of accomplishment and making me feel that I really have recovered well.

4. I could continue following the program and interacting with my doctor after surgery, making me feel that there was no distance from the doctor.
1. The calibration process for the sensors before exercise can be simplified.

2. Better to extend the battery standby time of the wearable sensors.

3. The app should use or allow a bigger font size.

4. The app could have pre-recorded exercise videos to help users understand more easily.

5. The elderly patients in particular took time to learn and get used to this device at the beginning. It is recommended to simplify the learning process.

6. Rewards could be offered to the users for accomplishing the daily exercise tasks and making them more fun.

Notes: Both feedback and suggestions are extracted from verbatim quotes.

\section{Discussion}

This study revealed that wearable IMU-based sensors can be used to trace the knee angular motion with acceptable reliability. The wearable motion sensors were integrated with interactive mobile apps and telecommunication technology to assist postoperative rehabilitation after TKA, which helped patients easily and correctly follow a daily HE program under the remote supervision of a physician. Compliance with the daily exercise regime was enhanced, thereby improving the recovery of knee extension angle and quadriceps muscle power, which led to a higher maximal and average angular velocity in 5TSST. 
Home-based rehabilitation programs are a popular choice for patients following TKA. Buhagiar et al. developed a post-TKA home program comprising general aerobic components as well as general functional and muscle-specific exercises; they demonstrated that the supervised home program was cost-effective and as efficient as inpatient rehabilitation in facilitating mobility in patients after TKA [12]. However, the success of their home-based program was based on clinician supervision. In practice, major obstacles for patients in following home-based physiotherapy are the absence of feedback by a physical therapist and the failure to remember or perform correct exercises $[27,28]$. The use of innovative methodologies, including wearable motion tracking devices has increased for assisting rehabilitation and monitoring activity at home in patients after joint replacement [29]. However, few clinical studies have supported the efficacy of these methods [30]. Kwasnicki RM et al. and Chiang $\mathrm{CY}$ et al. have demonstrated the feasibility of motion tracking sensors to monitor the recovery progress of ROM and kinematic profiles among patients following TKA [31,32]. However, unlike these studies using the MSD for functional assessment, our study integrated the MSD with a mobile phone-based system, which can provide an informative patient interface with comprehensible exercise instructions and support healthcare self-management by monitoring rehabilitation exercise performance. Furthermore, to strengthen the connection between physicians and patients, wireless telecommunication was used to synchronize data with the Doctor App to send information on each patient's progress, which enabled physicians to supervise rehabilitation remotely and provide instant feedback to patients. The MAR method increased patient adherence to the in-home exercise program, which was then reflected in the higher exercise completion rate and larger functional recovery after two months of training compared with patients in the HE group.

The ability to monitor knee angular motion and kinematic profile is another potential advantage of applying the MSD for in-home rehabilitation following TKA. The functional recovery of patients after TKA is conventionally measured using self-reported or physicianreported questionnaires. However, performance-based tests that can objectively assess the patient's actual ability to perform a task are seldom adopted in clinical practice following TKA. The kinematic and kinetic analyses of the sit-to-stand movement, which has been suggested to be an adequate performance-based measure in patients who received TKA [23,33], may have limited use because of the inaccessibility of the measuring equipment. However, the MSD used in this study can monitor the knee angular motion during in-home rehabilitation and indirectly trace the muscular, functional recovery based on the performance of angular velocity changes in 5TSST among patients after TKA. Wearable IMUs can be used to monitor the kinematic change of performance-based 5TSST remotely. In our study, patients in the MAR group displayed higher adherence to the in-home exercise program owing to the mobile phone-based informative interface and interaction with the physicians. Therefore, recovery of maximal and average angular velocity in 5TSST was better in the MAR group than in the HE group, even though the patient-reported WOMAC did not differ significantly between groups after two months of training. The IMU-based functional assessment helped physicians comprehensively and objectively follow the improvement in patients after TKA in addition to providing information on patient self-reported function.

The application of a wearable motion sensor device to improve patient compliance with in-home rehabilitation is promising, but some obstacles may need to be overcome. In addition to the stability of the sensor's application, a user-friendly app interface plays a vital role. The success of traditional clinical rehabilitation relies on robust patient-provider interaction [34]. Therefore, the new method of telerehabilitation using the MSD in postTKA rehabilitation programs should focus more on the connection between physicians and patients. In this study, the participants reported high scores for the user experience-related items of device helpfulness (mean 8.5 over10) and satisfaction with app design (mean 7.7 over 10), indicating high user satisfaction for the assistance of MSD in rehabilitation and the design of user interface. We believed that the Doctor and Patient app used in the MSD offered an informative and interactive interface, which is the key factor contributing to 
the excellent outcomes of the MSD-assisted rehabilitation and high user satisfaction in this feasibility study. However, the score for ease of operability reported by the participants was relatively low (mean 5.0 over 10), which may indicate the potential difficulty of using the MSD and, therefore, reflect on the ordinary score for the user's interest in future use (mean 5.8 over 10 ). In addition, the user experience feedback indicated that the complexity of the MSD design with a relatively steep learning curve could be further improved. For elderly users, simplifying the operability for the MSD will be a development target in the future.

Overall, the positive results obtained justify further work on the application of the MSD in telerehabilitation following joint replacement. The telemedicine industry in the orthopedic field is poised for growth in response to the 2019 coronavirus (COVID-19) pandemic [35]. An example of a successful telemedicine model is the application of virtual exercise rehabilitation in-home therapy, which can provide similar effects as traditional home or clinic physiotherapy and can significantly decrease 3-month healthcare costs after TKA [36]. In contrast to virtual physiotherapy, the MSD-assisted rehabilitation model presented in this study supports image-based and sensor-based telerehabilitation using an activity recognition model, an interactive 3D avatar in mobile phone applications, and a wireless telecommunicated network which enables more comprehensive physiotherapy for patients following TKA.

The main limitation of this study is the small sample size, which prevents this study from robust conclusions on the effectiveness of MSD-assisted home-based rehabilitation for patients following total knee replacement. However, the value of this study was to offer novel but preliminary results on the feasibility of the use of MSD in implications for post-TKA rehabilitation. We, therefore, recorded and presented the user experience from the participants in the MAR group, anticipating that this experience may facilitate the future development of MSD in telerehabilitation, especially in the post-TKA telerehabilitation scenario. Second, owing to the large national differences in post-TKA rehabilitation protocol, the protocol used in this trial, including the exercise contents, the different study time points, and the short follow-up period, may create potential bias resulting in difficulty in making conclusive statements. Third, we only recorded the postoperative pain score during follow-ups without documenting the analgesic use, which may mask the influence of pain on patient adherence to the training program. Fourth, the allocation of patients was not randomized because of their varying levels of familiarity with mobile apps and MSDs. Older adult patients may not favor new rehabilitation technology [37]. The gap in the acceptance of new technology among elderly patients may have caused a potential selection bias. The patients who accepted MSD-assisted rehabilitation and who were allocated to the MAR group were relatively highly educated, although no significant difference was noted. Furthermore, these patients may have had stronger motivation for rehabilitation training. Future studies should consider a training program for MSD usage before enrollment to prevent any potential selection bias. Finally, the four item questionnaire used in measurement of the user experience is not a standardized instrument, so that the questionnaire may carry the limitation of unidentified psychometric properties. Nevertheless, our data showed that the internal consistency of the four item questionnaire was excellent (Cronbach's alpha $=0.93$ ). Therefore, we believe that the measurement is reasonably validated for assessing user experience.

\section{Conclusions}

This study proved that wearable IMU-based sensors can be used to trace the knee angular motion with acceptable reliability. In addition, the application of a wearable motion sensor device in combination with interactive mobile apps is promising in the post-TKA telerehabilitation scenario. Although with the major limitation on the small number of participants, this preliminary study demonstrated that MSD-assisted home-based rehabilitation following TKA is a potentially useful treatment model of telerehabilitation because it enhances patient adherence to an in-home exercise program, which improves functional recovery. This model helps overcome critical obstacles in home-based physiotherapy for 
patients who have received TKA, which demonstrates the feasibility of MSD in telerehabilitation and has crucial implications for patients and health systems. Future investigations on the usability, cost-effectiveness and treatment efficacy of the MSD in telerehabilitation in large patient cohorts are warranted to demonstrate the viability of the MSD in real-world environments. However, the widespread use of the MSD in telerehabilitation for various musculoskeletal disorders is expected once the technology is fine-tuned.

Supplementary Materials: The following supporting information can be downloaded at: https: //www.mdpi.com/article/10.3390/app12052433/s1.

Author Contributions: Conceptualization, O.K.-S.L.; methodology, Y.-P.C.; investigation, Y.-P.C. and Y.-J.K.; resources, Y.-P.C. and Y.-J.K.; data curation, Y.-P.C. and C.-Y.L.; writing-original draft preparation, Y.-P.C.; writing-review and editing, O.K.-S.L.; visualization, O.K.-S.L.; supervision, O.K.-S.L.; project administration, O.K.-S.L.; funding acquisition, Y.-P.C. All authors have read and agreed to the published version of the manuscript.

Funding: The APC was funded by Taipei Medical University (Grant numbers TMU110-AE1-B07).

Institutional Review Board Statement: The Ethics Committee of Taipei Medical University approved the entire protocol and instrumentation TMU-JIRB N201802034. All participants consented to participation in the study and the publication of data.

Informed Consent Statement: Informed consent was obtained from all subjects involved in the study. Written informed consent has been obtained from the patient(s) to publish this paper.

Data Availability Statement: The data that support the findings of this study are available from the corresponding author upon reasonable request.

Acknowledgments: The authors acknowledge financial support by the "Development and Construction Plan" of the School of Medicine, National Yang-Ming University (107F-M01-0504) and Aiming for the Top University Plan, a grant from the Ministry of Education.

Conflicts of Interest: The authors declare no conflict of interest.

\section{References}

1. Llopart-Carles, N.; García-López, S.; Rejas-Gutierrez, J. Disability-adjusted life expectancy lost due to pain severity and usual analgesic treatment among older adults with osteoarthritis in Spain. Aging Clin. Exp. Res. 2020, 33, 1285-1295. [CrossRef] [PubMed]

2. Zhang, Y.; Jordan, J.M. Epidemiology of osteoarthritis. Clin. Geriatr. Med. 2010, 26, 355-369. [CrossRef] [PubMed]

3. Konopka, J.F.; Lee, Y.Y.; Su, E.P.; McLawhorn, A.S. Quality-Adjusted Life Years After Hip and Knee Arthroplasty: Health-Related Quality of Life After 12,782 Joint Replacements. JBJS Open Access 2018, 3, e0007. [CrossRef] [PubMed]

4. Inacio, M.C.S.; Paxton, E.W.; Graves, S.E.; Namba, R.S.; Nemes, S. Projected increase in total knee arthroplasty in the United States-An alternative projection model. Osteoarthr. Cartil. 2017, 25, 1797-1803. [CrossRef]

5. Artz, N.; Dixon, S.; Wylde, V.; Beswick, A.; Blom, A.; Gooberman-Hill, R. Physiotherapy provision following discharge after total hip and total knee replacement: A survey of current practice at high-volume NHS hospitals in England and wales. Musculoskelet. Care 2013, 11, 31-38. [CrossRef]

6. $\quad$ Mahomed, N.N.; Davis, A.M.; Hawker, G.; Badley, E.; Davey, J.R.; Syed, K.A.; Coyte, P.C.; Gandhi, R.; Wright, J.G. Inpatient compared with home-based rehabilitation following primary unilateral total hip or knee replacement: A randomized controlled trial. J. Bone Jt. Surg. Am. 2008, 90, 1673-1680. [CrossRef]

7. Kauppila, A.M.; Kyllönen, E.; Ohtonen, P.; Hämäläinen, M.; Mikkonen, P.; Laine, V.; Siira, P.; Mäki-Heikkilä, P.; Sintonen, H.; Leppilahti, J.; et al. Multidisciplinary rehabilitation after primary total knee arthroplasty: A randomized controlled study of its effects on functional capacity and quality of life. Clin. Rehabil. 2010, 24, 398-411. [CrossRef]

8. Artz, N.; Elvers, K.T.; Lowe, C.M.; Sackley, C.; Jepson, P.; Beswick, A.D. Effectiveness of physiotherapy exercise following total knee replacement: Systematic review and meta-analysis. BMC Musculoskelet. Disord. 2015, 16, 15. [CrossRef]

9. Moffet, H.; Collet, J.P.; Shapiro, S.H.; Paradis, G.; Marquis, F.; Roy, L. Effectiveness of intensive rehabilitation on functional ability and quality of life after first total knee arthroplasty: A single-blind randomized controlled trial. Arch. Phys. Med. Rehabil. 2004, 85, 546-556. [CrossRef]

10. Michard, F.; Gan, T.J.; Kehlet, H. Digital innovations and emerging technologies for enhanced recovery programmes. Br. J. Anaesth. 2017, 119, 31-39. [CrossRef]

11. Carvalho, E.; Bettger, J.P.; Goode, A.P. Insurance Coverage, Costs, and Barriers to Care for Outpatient Musculoskeletal Therapy and Rehabilitation Services. N. C. Med. J. 2017, 78, 312-314. [PubMed] 
12. Buhagiar, M.A.; Naylor, J.M.; Harris, I.A.; Xuan, W.; Kohler, F.; Wright, R.; Fortunato, R. Effect of Inpatient Rehabilitation vs a Monitored Home-Based Program on Mobility in Patients With Total Knee Arthroplasty: The HIHO Randomized Clinical Trial. JAMA 2017, 317, 1037-1046. [CrossRef] [PubMed]

13. Smith, T.; Withers, T.; Luben, R.; Sackley, C.; Jones, A.; MacGregor, A. Changes in physical activity following total hip or knee arthroplasty: A matched case-control study from the EPIC-Norfolk cohort. Clin. Rehabil. 2017, 31, 1548-1557. [CrossRef] [PubMed]

14. Bassett, S.F.; Prapavessis, H. Home-Based Physical Therapy Intervention With Adherence-Enhancing Strategies Versus ClinicBased Management for Patients With Ankle Sprains. Phys. Ther. 2007, 87, 1132-1143. [CrossRef]

15. Wang, Q.; Markopoulos, P.; Yu, B.; Chen, W.; Timmermans, A. Interactive wearable systems for upper body rehabilitation: A systematic review. J. Neuroeng. Rehabil. 2017, 14, 20. [CrossRef]

16. Ongvisatepaiboon, K.; Chan, J.H.; Vanijja, V. Smartphone-Based Tele-Rehabilitation System for Frozen Shoulder Using a Machine Learning Approach. In Proceedings of the 2015 IEEE Symposium Series on Computational Intelligence, Cape Town, South Africa, 7-10 December 2015; pp. 811-815.

17. Timmermans, A.A.; Seelen, H.A.; Geers, R.P.; Saini, P.K.; Winter, S.; te Vrugt, J.; Kingma, H. Sensor-based arm skill training in chronic stroke patients: Results on treatment outcome, patient motivation, and system usability. IEEE Trans. Neural Syst. Rehabil. Eng. 2010, 18, 284-292. [CrossRef]

18. Lemmens, R.J.; Janssen-Potten, Y.J.; Timmermans, A.A.; Smeets, R.J.; Seelen, H.A. Recognizing complex upper extremity activities using body worn sensors. PLoS ONE 2015, 10, e0118642. [CrossRef]

19. Filippeschi, A.; Schmitz, N.; Miezal, M.; Bleser, G.; Ruffaldi, E.; Stricker, D. Survey of Motion Tracking Methods Based on Inertial Sensors: A Focus on Upper Limb Human Motion. Sensors 2017, 17, 1257. [CrossRef]

20. Chen, Y.P.; Lin, C.Y.; Tsai, M.J.; Chuang, T.Y.; Lee, O.K. Wearable Motion Sensor Device to Facilitate Rehabilitation in Patients With Shoulder Adhesive Capsulitis: Pilot Study to Assess Feasibility. J. Med. Internet Res. 2020, 22, e17032. [CrossRef]

21. Open Source IMU and AHRS Algorithms. Available online: https:/ / x-io.co.uk/open-source-imu-and-ahrs-algorithms / (accessed on 4 January 2022).

22. Medina-Mirapeix, F.; Vivo-Fernández, I.; López-Cañizares, J.; García-Vidal, J.A.; Benítez-Martínez, J.C.; Del Baño-Aledo, M.E. Five times sit-to-stand test in subjects with total knee replacement: Reliability and relationship with functional mobility tests. Gait Posture 2018, 59, 258-260. [CrossRef]

23. Boonstra, M.C.; Schwering, P.J.A.; De Waal Malefijt, M.C.; Verdonschot, N. Sit-to-Stand Movement as a Performance-Based Measure for Patients With Total Knee Arthroplasty. Phys. Ther. 2010, 90, 149-156. [CrossRef] [PubMed]

24. Quintana, J.M.; Escobar, A.; Arostegui, I.; Bilbao, A.; Azkarate, J.; Goenaga, J.I.; Arenaza, J.C. Health-related quality of life and appropriateness of knee or hip joint replacement. Arch. Intern. Med. 2006, 166, 220-226. [CrossRef] [PubMed]

25. Flandry, F.; Hunt, J.P.; Terry, G.C.; Hughston, J.C. Analysis of subjective knee complaints using visual analog scales. Am. J. Sports Med. 1991, 19, 112-118. [CrossRef] [PubMed]

26. Landis, J.R.; Koch, G.G. The measurement of observer agreement for categorical data. Biometrics 1977, 33, 159-174. [CrossRef]

27. Sluijs, E.M.; Kok, G.J.; van der Zee, J. Correlates of exercise compliance in physical therapy. Phys. Ther. 1993, 73, 771-782; discussion 783-776. [CrossRef]

28. Escolar-Reina, P.; Medina-Mirapeix, F.; Gascón-Cánovas, J.J.; Montilla-Herrador, J.; Jimeno-Serrano, F.J.; de Oliveira Sousa, S.L.; del Baño-Aledo, M.E.; Lomas-Vega, R. How do care-provider and home exercise program characteristics affect patient adherence in chronic neck and back pain: A qualitative study. BMC Health Serv. Res. 2010, 10, 60. [CrossRef]

29. Toogood, P.A.; Abdel, M.P.; Spear, J.A.; Cook, S.M.; Cook, D.J.; Taunton, M.J. The monitoring of activity at home after total hip arthroplasty. Bone Jt. J. 2016, 98-b, 1450-1454. [CrossRef]

30. Bahadori, S.; Immins, T.; Wainwright, T.W. A review of wearable motion tracking systems used in rehabilitation following hip and knee replacement. J. Rehabil. Assist. Technol. Eng. 2018, 5. [CrossRef]

31. Kwasnicki, R.M.; Ali, R.; Jordan, S.J.; Atallah, L.; Leong, J.J.; Jones, G.G.; Cobb, J.; Yang, G.Z.; Darzi, A. A wearable mobility assessment device for total knee replacement: A longitudinal feasibility study. Int. J. Surg. 2015, 18, 14-20. [CrossRef]

32. Chiang, C.Y.; Chen, K.H.; Liu, K.C.; Hsu, S.J.; Chan, C.T. Data Collection and Analysis Using Wearable Sensors for Monitoring Knee Range of Motion after Total Knee Arthroplasty. Sensors 2017, 17, 418. [CrossRef]

33. Itokazu, M.; Uemura, S.; Aoki, T.; Takatsu, T. Analysis of rising from a chair after total knee arthroplasty. Bull. Hosp. Jt. Dis. 1998, 57, 88-92. [PubMed]

34. Oosterhof, B.; Dekker, J.H.; Sloots, M.; Bartels, E.A.; Dekker, J. Success or failure of chronic pain rehabilitation: The importance of good interaction-A qualitative study under patients and professionals. Disabil. Rehabil. 2014, 36, 1903-1910. [CrossRef] [PubMed]

35. Makhni, M.C.; Riew, G.J.; Sumathipala, M.G. Telemedicine in Orthopaedic Surgery: Challenges and Opportunities. J. Bone Jt. Surgery. Am. 2020, 102, 1109-1115. [CrossRef] [PubMed]

36. Prvu Bettger, J.; Green, C.L.; Holmes, D.N.; Chokshi, A.; Mather, R.C., 3rd; Hoch, B.T.; de Leon, A.J.; Aluisio, F.; Seyler, T.M.; Del Gaizo, D.J.; et al. Effects of Virtual Exercise Rehabilitation In-Home Therapy Compared with Traditional Care After Total Knee Arthroplasty: VERITAS, a Randomized Controlled Trial. J. Bone Jt. Surgery. Am. 2020, 102, 101-109. [CrossRef] [PubMed]

37. Axelrod, L.; Fitzpatrick, G.; Burridge, J.; Mawson, S.; Smith, P.; Rodden, T.; Ricketts, I. The reality of homes fit for heroes: Design challenges for rehabilitation technology at home. J. Assist. Technol. 2009, 3, 35-43. [CrossRef] 